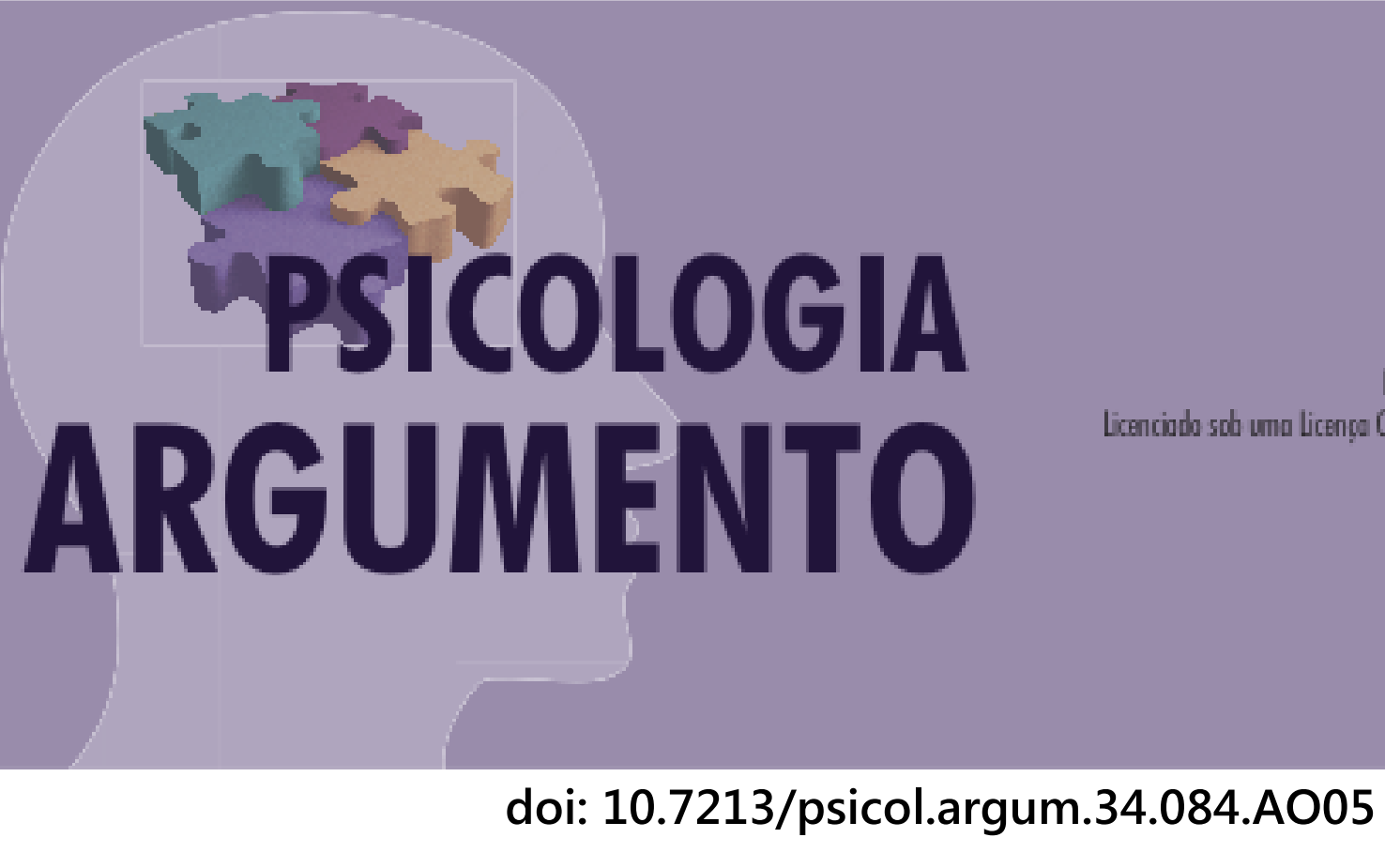

ISSN 01037013

ARGUMENTO

\title{
Habilidades sociais e bullying: um estudo entre agressores e vítimas
}

\author{
Social skills and bullying: a study between aggressors and vic- \\ tims
}

Zeimara de Almeida Santos ${ }^{[a]}$, Adriana Benevides Soares ${ }^{[b]}$

[a] Universidade Salgado de Oliveira, Niterói, Rio de Janeiro, Brasil - zeimaradealmeida@gmail.com

[b] Universidade Salgado de Oliveira, Niterói, Rio de Janeiro, Brasil - adribenevides@gmail.com

\section{Resumo}

Atitudes agressivas na escola constituem problemas que comprometem a aprendizagem e influenciam no desempenho escolar, perturbam as relações interpessoais e o desenvolvimento sócio emocional das crianças e jovens reduzindo o clima de segurança e proteção sentido por todos. A violência escolar está relacionada aos comportamentos agressivos e antissociais, incluindo os conflitos interpessoais, cujos tipos mais comuns de violência praticada por jovens são a violência, verbal, simbólica e o bullying. O fenômeno na escola é uma violência que tem sido associada a diversos comprometimentos no desenvolvimento de crianças e jovens, além de problemas psicológicos, pois se caracteriza como um comportamento agressivo, cruel, intencional, de forma repetitiva e sem limites. Pode-se dizer que comportamentos socialmente competentes são preventivos de problemas de comportamento, incluindo o bullying. O estudo se propôs identificar relações entre habilidades sociais e problemas de comportamento em vítimas e agressores de 
bullying. Participaram do estudo cerca de 400 estudantes do $6^{\circ}$ ano do Ensino Fundamental, provenientes de escolas públicas e privadas da região metropolitana do Rio de Janeiro. Os principais resultados mostraram que habilidades sociais se correlacionam negativamente com problemas de comportamento e que não houve diferença quanto as Habilidades Sociais nos envolvidos no bullying. Sendo assim, para amostra total, quanto mais problemas de comportamento os indivíduos apresentam menor o escore de habilidades sociais. O estudo em questão contribui com dados empíricos e discussões acerca do bullying em situação escolar.

Palavras-chave: bullying, violência, habilidades sociais, problemas de comportamento.

\begin{abstract}
Aggressive attitudes in school are problems that hinder learning and interfere with school performance, disrupts interpersonal relationships and socio-emotional development of children and young people, reducing the climate of security felt by all. School violence is related to aggressive and antisocial behavior, including interpersonal conflicts, whose most common types of violence among young people is violence, verbal, symbolic and bullying. The phenomenon in school is a violence that has been linked to various problems in the development of children and youth, characterized as aggressive, cruel, intentional, repetitive and without limits. You could say that socially competent behaviors are preventive, including bullying. The study aimed to identify the relationship between social skills and problem behavior in victims and bullies of bullying. Study participants were 400 students from the 6th grade of elementary school, from public and private schools in the metropolitan area of Rio de Janeiro. The main results showed that social skills are negatively related to behavior problems and that there was no difference in social skills of those involved in bullying. So for the total sample, individuals with more behavior problems have less social skills. This study provides empirical data and discussions about bullying in the school situation.
\end{abstract}

Keywords: bullying, violence, social skills, behavior problems.

\title{
Introdução
}

A violência que ocorre em ambientes escolares é caracterizada por comportamentos agressivos e antissociais, como conflitos interpessoais. O bullying pode ser compreendido como um subconjunto e formas de comportamentos agressivos, verbais ou físicos, intencionais e repetitivos, ocorrendo sem uma motivação evidente. Pode ser realizado por um ou mais estudantes contra outros, causando dor e angústia e é executado dentro de uma relação desigual de poder (Peterson, 2009). Apresenta-se como um problema de ordem social tanto para o Brasil como para diversos outros países. No Brasil, o levantamento realizado por Malta et al. (2010) com 60.973 escolares de 1.453 escolas públicas e privadas das 26 capitais brasileiras e do Distrito Federal apontou que 5,4\% dos estudantes entrevistados responderam ter sofrido bullying quase sempre ou sempre nos últimos 30 dias e $25,4 \%$ dos respondentes foram raramente ou às vezes vítimas de bullying. Os dados internacionais obtidos através do Relatório Internacional da Organização Mundial de Saúde apontam o bullying como um problema mundial que afeta em média $13 \%$ dos alunos em idade escolar de 11 anos (Matos, 2012). 
As experiências de bullying tendem a aumentar entre as idades de 11 a 12 anos (Organização Mundial de Saúde, 2009), idade escolar especificamente do sexto ano do Ensino Fundamental. Sendo assim, o presente estudo escolheu o sexto ano por se tratar de um período de mudança fisiológica, emocional e social entre a infância e a adolescência e também por ser um período de transição no currículo escolar (Wiest \& Lechner, 2013). O ingresso do estudante no sexto ano do Ensino Fundamental traz, com frequência, problemas de adaptação (Moura, Cruz \& Quevedo, 2011), pois o estudante encontra um ambiente escolar com características sociais e emocionais que muitas vezes não está acostumado. Os professores são especialistas, trabalham em várias instituições de ensino, o que dificulta uma relação individual mais à próxima do aluno (Moura, Cruz, \& Quevedo, 2011). Para Wiest e Lechner (2013) o sexto ano tem sido apresentado como um período de instabilidade, cuja metodologia, conteúdo e relacionamento com professor - aluno sofre alterações. Caracteriza-se como um período de transição de unidocente para pluridocente que é marcada pela saída de um segmento para outro do Ensino Fundamental. Para Cainelli e Oliveira (2012) os alunos demonstram tensão no que se refere à mudança de ambiente, espaço, reestruturação das disciplinas e interação alunos e professores. A transição escolar no início da adolescência é preocupante porque coincide com o início da puberdade e com as mudanças de contexto que podem tornar este período particularmente desafiador (Wiest \& Lechner, 2013). Gresham (2009) complementa argumentando que diante de tais transformações e desafios que esta faixa etária requer é possível encontrar uma possível crise de ruptura levando a transtornos e sofrimentos. Essa ruptura acarreta déficits no desempenho acadêmico e social, causando muitas vezes o uso inadequado de comportamentos antissociais, aumentando o grau de ansiedade, agressividade, medo e angústias diante de situações novas.

A infância e a adolescência são períodos nos quais a aprendizagem e a convivência social se solidifica e podem ser identificadas como fases do desenvolvimento em que os indivíduos são vulneráveis. São momentos críticos para o aprendizado de habilidades sociais e o manejo de sentimentos (Z. Del Prette \& Del Prette, 2009). De acordo com Z. Del Prette e Del Prette (2009), uma criança que tem a oportunidade de aprender e desenvolver o repertório de HS tende a apresentar uma melhor adaptação e ajustamento social como também uma maior satisfação nas interações com outras pessoas. Nesse sentido Z Del Prette e Del Prette (2009) explicam que déficits de habilidades sociais em etapas formativas da vida do indivíduo, podem comprometer fases posteriores de seu ciclo vital, visto problemas psicológicos, como a delinquência juvenil por exemplo. Desta forma a promoção das habilidades sociais tem sido apontada como comportamentos protetivos frente aos problemas de comportamento principalmente no que diz respeito à violência escolar. Pesquisas na área de relações interpessoais vêm confirmando uma associação entre déficit de habilidades sociais, problemas de comportamento e sociopatias em geral (Z. Del Prette \& Del Prette, 2009).

Os problemas comportamentais e emocionais que acompanham os transtornos psicológicos podem envolver agressividade física e/ou verbal, depressão, isolamento social, ansiedade e fobia social. As dificuldades interpessoais que caracterizam esses problemas decorrem de um repertório pobre de habilidades sociais. Este é o caso da empatia, impulsividade, baixa autoestima, entre outros (Z. Del Prette \& Del Prette, 2009). As demandas de cada ciclo vital e as respostas apresentadas pelo indivíduo revelam a aquisição de comportamentos sociais envoltos em um processo de aprendizagem ao longo da vida. Garner e 
Hinton (2010) entendem que na observação que as crianças fazem dos comportamentos sociais de seus pais; passam a imitá-los. Nesse sentido, a qualidade dos relacionamentos de uma criança na escola, tem como base os recursos comportamentais adquiridos no contexto familiar, ampliando ou reduzindo oportunidades de desenvolvimento de habilidades sociais. Sendo a educação uma prática social, é de grande relevância a qualidade das interações sociais dentro do contexto escolar, sobretudo no aperfeiçoamento do processo educacional e no desenvolvimento socioemocional.

As habilidades sociais (HS) vêm sendo reconhecidas, de forma mais generalizada, para todas as crianças, como um componente crucial para a aprendizagem e o sucesso escolar, bem como para o desenvolvimento socioemocional e o ajustamento na escola ( $Z$. Del Prette \& Del Prette, 2009). As HS acontecem de alguma forma para designar um conjunto de capacidades comportamentais aprendidas que envolvem interações sociais associadas ao comportamento (Z. Del Prette \& Del Prette, 2009). Pode-se dizer que são protetivas de problemas de comportamento, incluindo o bullying, possibilitando assim uma articulação entre práticas socioeducativas adequadas e o desenvolvimento da criança (Z. Del Prette \& Del Prette, 2009).

Em geral, os problemas de comportamento expressam uma necessidade, medo ou mesmo uma tentativa de evitar algo desagradável. Carpenter e Fergunson (2011) declaram que atitudes hostis que violam o direito a integridade física e psicológica do indivíduo, com o intuito de causar sofrimento, caracterizam o bullying. Os problemas de comportamento podem consistir no fato de uma pessoa se tornar agressiva, ameaçadora, apática, hiperativa ou retraída (Bandeira \& Hutz, 2012). Del Prette, Barreto e Freitas (2012) fizeram um estudo com o objetivo de avaliar as HS na comorbidade entre problemas de comportamento e dificuldade de aprendizagem em crianças de oito a doze anos de idade. Em uma amostra de 50 crianças de ambos os sexos. Foram encontrados resultados que indicaram déficits de aquisição de desempenho nas HS das crianças, em especial nas habilidades assertivas e empáticas, o que justifica o comprometimento com o desenvolvimento socioemocional. Desta forma, A. Del Prette e Del Prette (2010) argumentam que o bom desempenho nas interações sociais requer habilidades sociais. Durante situações de interação social, pode-se dizer que as emoções contribuem para o desenvolvimento de HS, se estabelece um padrão adequado de comunicação que prevê menor probabilidade de se apresentar problemas de comportamento. Assim, o presente estudo objetivou verificar se existe associação entre habilidades sociais e problemas de comportamento em agressores e vítimas de bullying. Foram investigadas as seguintes hipóteses 1) Habilidades sociais se correlacionam negativamente com problemas de comportamento para todos os estudantes da amostra; 2) Vítimas e agressores no contexto do bullying apresentam menos habilidades sociais do que os que não estão envolvidos no contexto de bullying; 3) Agressores (bullies) apresentam mais problemas de comportamento do que as vítimas.

\section{Método}




\section{Participantes}

Participaram do estudo 400 estudantes de 3 instituições públicas e 3 privadas do interior do estado do Rio de Janeiro. A amostra é não probabilística de conveniência, os alunos estão incluídos em uma faixa etária entre 11 e 13 anos de ambos os sexos $(\mathrm{M}=11,58$ e $\mathrm{DP}=0,77)$ sendo $47 \%$ meninos e $53 \%$ meninas do sexto ano do Ensino Fundamental.

\section{Instrumentos}

Os instrumentos utilizados na seleção da amostra e na coleta de dados foram à Escala de Problemas de Comportamento para Estudantes e a Escala de Habilidades Sociais do Sistema de Avaliação de Habilidades Sociais (SSRS) (Gresham \& Elliott, 1990) e validado para o Brasil por Bandeira, Z. Del Prette, Del Prette e Magalhães, (2009) e o Questionário de Bullying de Olweus (1989) adaptado por Barbosa (2009) para o português brasileiro.

O SSRS na versão de autoavaliação dos estudantes é composta de 34 itens, distribuídos em seis componentes: Responsabilidade (composto de sete itens e tem Alpha de Cronbach $=0,62)$; reúne itens relativos a comportamentos que demons $\neg$ tram compromisso com as tarefas e com as pessoas no ambiente escolar, dentre elas seguir uma rotina diária, deixar o ambiente limpo e arrumado, ter cuidado com a organização do seu material e usar adequadamente o tempo livre Empatia (engloba quatro itens e tem Alpha de Cronbach = $0,51)$; agrupa itens sobre comportamentos que demonstram inte $\neg$ resse, respeito e preocupação com os colegas e demais pessoas, entender os colegas quando estão irritados ou infelizes por alguma situação, ouvir os problemas dos amigos, se colocar no lugar do outro quando acontece algo desagradável com as pessoas, tentar resolver problema ou briga de colegas; Assertividade (composto de sete itens e tem Alpha de Cronbach $=0,58$ ); reúne itens sobre comportamentos que questionam regras injustas, autocontrole emocional ao lidar com discussões de classe e ao discordar de adultos, cumprimentar pessoas e emitir opiniões; Autocontrole (engloba quatro itens e tem Alpha de Cronbach $=0,46$ ); itens sobre comportamentos que demonstram domínio sobre as próprias reações emocionais em situações de conflitos, pedir e esperar permissão para usar coisas de outros, de permanecer ouvindo as pessoas que estão falando, de controlar raiva quando zangado; Evitação de Problemas (com seis itens e tem Alpha de Cronbach $=0,49$ ); composto de itens sobre comportamentos que demonstram domínio sobre as próprias reações emocionais, tais como: fazer provocações, ignorar colegas fazendo brincadeiras, solicitar e esperar que os colegas o (a) aceitem em brincadeira ou jogo, não concordar com as ideias dos adultos sem briga ou discussão, resolver problemas ou brigas argumentando com o colega; Expressão de Sentimento Positivo (composto de quatro itens e tem Alpha de Cronbach = 0,49 ); compreende itens que representam comportamen $\neg$ tos que expressam aprovação aos comportamentos dos demais, expressando sentimentos positivos, por exemplo: elogiar e cumprimentar amigos e demais pessoas, expressar carinho dizendo que gosta dos amigos, dizer a um adulto que aprecia sua atitude. Para a escala global, a análise da consistência interna teve um Alfa de Cronbach de 0,78, bem como correlações significativas itemescore total.

O Questionário de Bullying foi adaptado primeiramente por Pereira (2008) para o português de Portugal e posteriormente por Barbosa para o português brasileiro em 2009. Conforme evidencias de validade do questionário para o contexto brasileiro que foi apre- 
sentado por Lamas, Freitas e Barbosa (2013) trata-se de uma medida com 42 questões divididas em quatro sessões de caracterização sócia demográfica composto de 15 itens (diz respeito a dados pessoais do respondente procurando obter informação sobre o gênero da criança/jovem, a idade, a profissão e habilitações escolares dos pais, bem como a zona de residência); ser Vítima de bullying composto de 13 itens e Alfa de Cronbach de 0,40 (este bloco é relativo aos pares, nomeadamente quem são os melhores amigos da criança/jovem, qual a frequência da ocorrência que foi vitimada, onde a frequência é questionada; os tipos; os locais em que ocorrem os maus-tratos; e quem pratica a agressão); ser Agressor composto de 04 itens e Alfa de Cronbach de 0,60 (este bloco é questionado sobre a prática de agressão a outros no trajeto para a escola, se já se juntou a outras crianças para agredir alguém e a frequência com que isso acontece. É ainda questionado o número de alunos da sua sala que, na sua opinião, agridem outros) e Recreios composto de 10 itens (avalia o gosto que a criança tem pelo recreio, se tem espaço para desenvolver as suas brincadeiras e o que pensa dos recreios).Para se considerar vítima, o aluno deve ter sido agredido por um ou mais pares com essa mesma frequência num intervalo de três meses e para se considerar agressor, o estudante deve ter agredido um ou mais colegas por três ou mais vezes num intervalo também de três meses. O Questionário de Bullying no estudo de Barbosa (2009) apresenta uma consistência interna $\alpha=0,80$.

\section{Procedimentos de Coletas de Dados}

Os questionários foram aplicados por vezes de forma coletiva e por vezes de forma individual nas respectivas escolas em horário escolar. A coleta foi realizada pelo pesquisador, com a presença do professor em sala de aula. Não houve controle de tempo para a resposta dos instrumentos, porém foi seguida uma ordem para aplicação dos questionários: Escala de Habilidades Sociais do Sistema de Avaliação de Habilidades Sociais para os pais, em seguida para os estudantes e o Questionário de Bullying.

\section{Procedimentos Éticos}

A presente pesquisa foi aprovada pelo Comitê de Ética sob o número processo 561.486/2014 em 25/02/2014. Foi solicitado por meio de carta de anuência, autorização das instituições de Ensino. Todos os responsáveis dos participantes assinaram Termo de Consentimento Livre e Esclarecido (TCLE) que informava os objetivos da pesquisa e as regras de preservação do anonimato. O TCLE também informava o contato do pesquisador caso fosse necessário e garantia o direito de retirada de seu consentimento a qualquer momento do estudo, segundo a orientação da Resolução 466/2012 do Conselho Nacional de Saúde.

\section{Procedimentos de análise de dados}

Os dados foram analisados utilizando o programa SPSS 20.0. Para analisar as relações entre os construtos foi realizado teste $t$ de Student para comparar as habilidades sociais com problemas de comportamento. Considerou-se nas comparações o nível de significância de $p \leq 0,05$. Para verificar as relações entre habilidades sociais e problemas de comportamento, utilizou-se o teste de correlação linear de Pearson. Para verificar se agressores (bullies) apresentam mais problemas de comportamento do que as vítimas utilizou-se o teste U de Mann-Whitney. 


\section{Apresentação e Discussão dos Resultados}

A primeira hipótese (Habilidades sociais se correlacionam negativamente com problemas de comportamento para todos os estudantes da amostra) foi confirmada, pois os resultados evidenciaram correlações negativas e fracas (Problema de Comportamento Briga se correlacionou com HS Responsabilidade, Empatia, Assertividade, Autocontrole da agressividade, Evitação de Problemas e Expressão de sentimento positivo; enquanto Problema de Comportamento Autoestima se correlacionou com HS Empatia).

Tabela 1. Correlação entre problemas de comportamento e habilidades sociais de estudantes $(N=400)$

\begin{tabular}{|c|c|c|c|c|c|c|c|c|c|c|}
\hline & & $\begin{array}{l}\text { PC } \\
\text { inquietação }\end{array}$ & $\begin{array}{l}\text { PC } \\
\text { briga }\end{array}$ & $\begin{array}{l}\mathrm{PC} \\
\text { autoestima }\end{array}$ & Responsab. & Empatia & Assertividade & Autocontrole & Evitação & $\begin{array}{l}\text { Expressão } \\
\text { outras }\end{array}$ \\
\hline PCInquietação & $\mathbf{r}$ & 1 & & & & & & & & \\
\hline \multirow{2}{*}{ PCBriga } & $\mathrm{r}$ & 0,18 & 1 & & & & & & & \\
\hline & $\mathrm{p}$ & 0,00 & & & & & & & & \\
\hline \multirow{2}{*}{ PCAutoestima } & $\mathrm{r}$ & 0,05 & 0,47 & 1 & & & & & & \\
\hline & $\mathrm{p}$ & 0,28 & 0,00 & & & & & & & \\
\hline \multirow{2}{*}{ Responsabilidade } & $\mathrm{r}$ & $-0,04$ & $-0,13$ & $-0,06$ & 1 & & & & & \\
\hline & $\mathrm{p}$ & 0,38 & 0,00 & 0,23 & & & & & & \\
\hline \multirow{2}{*}{ Empatia } & $\mathrm{r}$ & $-0,01$ & $-0,17$ & $-0,18$ & 0,26 & 1 & & & & \\
\hline & $\mathrm{p}$ & 0,78 & 0,00 & 0,00 & 0,00 & & & & & \\
\hline \multirow{2}{*}{ Assertividade } & $\mathrm{r}$ & $-0,01$ & $-0,14$ & $-0,08$ & 0,43 & 0,31 & 1 & & & \\
\hline & $\mathrm{p}$ & 0,83 & 0,00 & 0,11 & 0,00 & 0,00 & & & & \\
\hline \multirow{2}{*}{ Autocontrole } & $\mathrm{r}$ & $-0,01$ & $-0,13$ & $-0,02$ & 0,31 & 0,26 & 0,41 & 1 & & \\
\hline & $\mathrm{p}$ & 0,82 & 0,02 & 0,76 & 0,00 & 0,00 & 0,00 & & & \\
\hline \multirow{2}{*}{ Evitação } & $\mathrm{r}$ & $-0,03$ & $-0,16$ & $-0,05$ & 0,39 & 0,33 & 0,45 & 0,35 & 1 & \\
\hline & $\mathrm{p}$ & 0,56 & 0,00 & 0,245 & 0,00 & 0,00 & 0,00 & 0,00 & & \\
\hline \multirow{2}{*}{ Expressão } & $\mathrm{r}$ & 0,06 & $-0,18$ & $-0,09$ & 0,32 & 0,37 & 0,40 & 0,30 & 0,43 & 1 \\
\hline & $\mathrm{p}$ & 0,23 & 0,00 & 0,05 & 0,00 & 0,00 & 0,00 & 0,00 & 0,00 & \\
\hline Outras & $r$ & $-0,18$ & $-0,00$ & 0,02 & 0,29 & 0,21 & 0,33 & 0,29 & 0,38 & 0,27 \\
\hline
\end{tabular}

Cia e Costa (2012) também realizaram um estudo relacionando problemas de comportamento e habilidades sociais com 215 alunos do Ensino Fundamental cujo resultado indicou que quanto mais elaboradas são as habilidades sociais menores são os índices de problemas de comportamento. A ocorrência de comportamentos problemáticos obtidos no presente estudo se relacionou negativamente com a habilidade Responsabilidade visto que quando os alunos não demonstram compromisso com suas tarefas escolares, apresentam predisposição a comportamentos agressivos. De acordo com estudos de Carpenter e Ferguson (2011) que reflete sobre a questão de escolas numerosas, constatou-se que estudantes de escolas maiores apresentavam menor número de participação em atividades e assumiam menos responsabilidades, colaboravam menos e costumavam demonstrar comportamentos agressivos e retraídos. $\mathrm{O}$ estudo em questão apresentou relação também entre 
habilidade Empática e problema de comportamento de Briga, portanto quanto menos se preocupam com os colegas, mais ameaçam os outros alunos. Barreto, Freitas e Del Prette (2011), realizaram um estudo com 50 crianças que apresentavam problemas de comportamento e os resultados apontaram um déficit em habilidades sociais, sobretudo no que tange habilidades empáticas. Percebe-se ainda no presente estudo relação entre comportamento problemático e habilidade Assertiva, pois ao discordar dos adultos e questionar regras injustas os estudantes podem se mostrar agressivos. Essa capacidade de "dizer não" indica um modo de interagir de acordo com aquilo que se sente e que se percebe como sendo a conduta adequada a ser realizada (Cia, \& Costa, 2012), entretanto muitas vezes o comportamento que deveria ser assertivo acaba sendo agressivo, portanto não competente socialmente. Observou-se ainda correlação entre a habilidade de Autocontrole e problema de comportamento de Briga, no que diz respeito ao domínio sobre as reações emocionais em situações de conflito e controle da raiva quando zangado. É válido ressaltar que a baixa capacidade de autocontrole pode estar atrelada a comportamentos impulsivos, entre eles manifestações de violência (Bandeira, 2009; Marchezini-Cunha, \& Tourinho, 2010). Os resultados do estudo em questão demonstraram ainda a correlação entre a habilidade de Evitação de Problema com problema de comportamento de Briga, pois ao ignorar as provocações dos colegas discussões acontecem. O estudo de Vanderbilt e Augustyn (2010) verificou a associação entre habilidades sociais de crianças e bullying, assim diferenças significativas foram encontradas entre o subgrupo Evitação de Problemas e o escore geral. Evidenciando assim que as vítimas apresentaram escores mais altos de habilidades sociais de Evitação de Problemas que o grupo de não envolvidos no fenômeno.

Foi associada à classe de habilidade de Expressão de Sentimento Positivos e comportamento problemático de Briga, pois os comportamentos expressam a exteriorização de sentimentos positivos ao reagir inapropriadamente à pressão. Percebe-se então que a partir de uma convivência diária com os colegas de classe ocorre uma demanda de desempenhos complexos que podem facilitar ou dificultar o desenvolvimento de situações relacionais (Bandeira, Z. Del Prette, Del Prette, \& Magalhães, 2009).

Ainda para primeira a hipótese foi encontrada a relação entre a habilidade de Empatia e o comportamento problemático de Autoestima, indicando que quanto mais se reconhece as diferenças do colega e se colocar no lugar do outro, menos se consegue se expressar de forma adequada, assim o estudante prefere se isentar frente algumas situações. Com base nesses resultados Z. Del Prette e Del Prette (2009) destacam que a comunicação empática pode recuperar ou aumentar a autoestima entre as pessoas. Comportamentos agressivos na escola se constituem em dificuldades na aprendizagem que influenciam e perturbam as relações interpessoais e o desenvolvimento sócio emocional das crianças, reduzindo assim a percepção de segurança e proteção. No que tange esses comportamentos agressivos Thornberg (2010) afirmaram que os mesmos acontecem com maior frequência em escolas com alta rotatividade de docentes, inadequada supervisão pedagógica, organização ineficaz e metodologia incoerente. $\mathrm{O}$ bullying tende a desaparecer em ambientes onde existe um clima afetivo e de confiança entre as pessoas.

Na segunda hipótese do estudo sobre a diferença nos escores de habilidades sociais de vítimas e agressores e de estudantes não envolvidos no bullying foi realizado o teste $\mathrm{t}$ de Student para amostras independentes. Os resultados indicam não haver diferença entre os grupos testados. 
Tabela 2. Comparação das HS entre alunos envolvidos e não envolvidos no bullying

\begin{tabular}{|c|c|c|c|c|c|}
\hline Variável & Estudantes & Média & Desvio Padrão & $t$ & $P$ \\
\hline \multirow{2}{*}{ Responsabilidade } & envolvidos & 0,10 & 0,06 & 1.753 & 0,080 \\
\hline & não envolvidos & 0,10 & 0,05 & 1.963 & 0,060 \\
\hline \multirow{2}{*}{ Empatia } & envolvidos & 0,07 & 0,09 & 0.857 & 0,392 \\
\hline & não envolvidos & 0,07 & 0,09 & 0.822 & 0,418 \\
\hline \multirow{2}{*}{ Assertividade } & envolvidos & $-0,02$ & 0,07 & -0.215 & 0,830 \\
\hline & não envolvidos & $-0,02$ & 0,06 & -0.251 & 0,803 \\
\hline \multirow{2}{*}{ Autocontrole } & envolvidos & $-0,15$ & 0,08 & -1.827 & 0,068 \\
\hline & não envolvidos & $-0,15$ & 0,09 & -1.797 & 0,083 \\
\hline \multirow{2}{*}{ Evitação } & envolvidos & $-0,09$ & 0,07 & -1.227 & 0,221 \\
\hline & não envolvidos & $-0,09$ & 0,07 & -1.246 & 0,223 \\
\hline \multirow{2}{*}{ Expressão } & envolvidos & $-0,07$ & 0,08 & -0.909 & 0,364 \\
\hline & não envolvidos & $-0,07$ & 0,07 & -0.985 & 0,333 \\
\hline \multirow{2}{*}{ Outras } & envolvidos & 0,05 & 0,09 & 0.619 & 0,536 \\
\hline & não envolvidos & 0,05 & 0,08 & 0.666 & 0,511 \\
\hline \multirow{2}{*}{ Responsabilidade } & envolvidos & 0,10 & 0,06 & 1.753 & 0,080 \\
\hline & não envolvidos & 0,10 & 0,05 & 1.963 & 0,060 \\
\hline \multirow{2}{*}{ Empatia } & envolvidos & 0,07 & 0,09 & 0.857 & 0,392 \\
\hline & não envolvidos & 0,07 & 0,09 & 0.822 & 0,418 \\
\hline \multirow{2}{*}{ Assertividade } & envolvidos & $-0,01$ & 0,07 & -0.215 & 0,830 \\
\hline & não envolvidos & $-0,02$ & 0,06 & -0.251 & 0,803 \\
\hline \multirow{2}{*}{ Autocontrole } & envolvidos & $-0,15$ & 0,08 & -1.827 & 0,068 \\
\hline & não envolvidos & $-0,15$ & 0,06 & -1.797 & 0,083 \\
\hline \multirow{2}{*}{ Evitação } & envolvidos & $-0,09$ & 0,07 & -1.227 & 0,221 \\
\hline & não envolvidos & $-0,09$ & 0,07 & -1.246 & 0,223 \\
\hline \multirow{2}{*}{ Expressão } & envolvidos & $-0,07$ & 0,08 & -0.909 & 0,364 \\
\hline & não envolvidos & $-0,07$ & 0,06 & -0.985 & 0,333 \\
\hline \multirow{2}{*}{ Outras } & envolvidos & 0,05 & 0,09 & 0.619 & 0,536 \\
\hline & não envolvidos & 0,05 & 0,08 & 0.666 & 0,511 \\
\hline \multirow{2}{*}{ Responsabilidade } & envolvidos & 0,10 & 0,06 & 1.753 & 0,080 \\
\hline & não envolvidos & 0,10 & 0,05 & 1.963 & 0,060 \\
\hline \multirow{2}{*}{ Empatia } & envolvidos & 0,07 & 0,09 & 0.857 & 0,392 \\
\hline & não envolvidos & 0,07 & 0,09 & 0.822 & 0,418 \\
\hline \multirow{2}{*}{ Assertividade } & envolvidos & $-0,01$ & 0,07 & -0.215 & 0,830 \\
\hline & não envolvidos & $-0,01$ & 0,06 & -0.251 & 0,803 \\
\hline \multirow{2}{*}{ Autocontrole } & envolvidos & $-0,15$ & 0,08 & -1.827 & 0,068 \\
\hline & não envolvidos & $-0,15$ & 0,08 & -1.797 & 0,083 \\
\hline
\end{tabular}




\begin{tabular}{llllll}
\multirow{2}{*}{ Evitação } & envolvidos & $-0,09$ & 0,07 & -1.227 & 0,221 \\
& não envolvidos & $-0,09$ & 0,07 & -1.246 & 0,223 \\
\hline
\end{tabular}

O resultado apontou que não foi possível verificar diferença nas habilidades sociais, obtendo valores dentro da média esperada em suas habilidades. Vanderbilt e Augustyn (2010) afirmam que os agressores podem apresentar um bom repertório de habilidades sociais, já Peterson (2009) sugerem que crianças com intuito de serem aceitas, mas que pertencem a um grupo com características agressivas tendem a esconder o seu comportamento intimidador perante seus pares, através da repressão de seus próprios membros como alguém que não é desejável dentro do grupo e assim reforçar a sua identidade de coesão. De modo geral, a atenção das pesquisas sobre as habilidades sociais de indivíduos envolvidos no processo de bullying focaliza a compreensão de diversas habilidades, sem que se observe um grupo de habilidades específicas. Com o mesmo intuito de questionar o papel das habilidades sociais no bullying, Hamiwka et al. (2011) realizaram um estudo que comparou as habilidades sociais de crianças americanas entre oito e dezesseis anos de idade, vítimas e não-vítimas de bullying com quadro de epilepsia. Estendendo a discussão sobre o fenômeno, Gini, Pozzoli e Hauser (2011) relatam que os agressores, assim como as vítimas e testemunhas, podem ter conhecimentos dos valores morais, mas a diferença está nas dificuldades de compaixão moral ao que expressam vítimas e testemunhas. Os alvos de bullying sofrem com vergonha, medo, ansiedade e até mesmo se consideram merecedores dos maus tratos recebidos devido ao grande comprometimento de sua autoestima (Carpenter \& Ferguson, 2011). Na maioria das vezes, as vítimas não solicitam ajuda por possuírem sentimentos de insegurança e não reagirem ao bullying, podendo causar danos no rendimento escolar, recusar comparecer à escola, simular doenças, aspirar troca de colégio ou mesmo abandonar os estudos (Moura et al., 2011).

Os agressores geralmente manifestam pouca empatia, possuem na maioria das vezes família desestruturada afetivamente, possuem frequentemente também responsáveis que oferecem comportamentos violentos para solucionar os conflitos (Oliveira \& Barbosa, 2012). Para Vanderbilt e Augustyn (2010), os autores de bullying buscam alvo mais vulnerável para transferir agressões sofridas.

Os resultados evidenciaram que os estudantes mais velhos (12 e 13 anos) apresentaram mais chances de serem agressores em relação aos mais novos (11 anos). Estudos realizados nos Estados Unidos (Centers for Disease Control and Prevention, 2011) também apontaram maior prevalência de agressores entre os discentes de 13 a 16 anos em comparação aos de 11 e 12 anos. Isto se deve possivelmente porque os discentes mais novos tornam-se vítimas dos mais velhos, aumentando o número de agressores com o avanço da idade (Calbo, Busnello, Rigoli, \& Kristensen, 2009). Também nesse período entre as idades estudadas a aparência física está associada às agressões (Brixval, Rayce, Rasmussen, Holstein, \& Due, 2012).

Os resultados encontrados para a terceira hipótese são provenientes do teste para amostras pareadas entre agressores e vítimas. A análise identificou um grupo de agressores reduzido, razão pela qual foi utilizado um teste não paramétrico U de Mann-Whitney para a comparação entre os grupos. Verificou-se que não houve diferença estatística significativa entre as vítimas e agressores em todas as variáveis comparadas. 
Tabela 3. Comparação entre vítimas e agressores no que se refere aos problemas de comportamento sob a ótica dos estudantes

\begin{tabular}{|c|c|c|c|c|c|}
\hline & Envolvidos & $\mathrm{N}$ & Posto médio & \multicolumn{2}{|c|}{ Soma dos postos } \\
\hline & Vítima & 19 & 13,95 & & 265,00 \\
\hline \multirow[t]{3}{*}{ Inquietação } & Agressor & 6 & 10,00 & & 60,00 \\
\hline & Total & 25 & & & \\
\hline & Vítima & 19 & 12,87 & & 244,50 \\
\hline \multirow[t]{3}{*}{ Briga } & Agressor & 6 & 13,42 & & 80,50 \\
\hline & Total & 25 & & & \\
\hline & Vítima & 19 & 11,74 & & 223,00 \\
\hline \multirow[t]{3}{*}{ Autoestima } & Agressor & 6 & 17,00 & & 102,00 \\
\hline & Total & 25 & & & \\
\hline & & & Inquietação & Briga & Autoestima \\
\hline \multicolumn{2}{|l|}{ Mann-Whitney U } & & 39,00 & 54,50 & 33,00 \\
\hline \multicolumn{2}{|l|}{ Wilcoxon W } & & 60,00 & 244,50 & 223,00 \\
\hline \multicolumn{2}{|l|}{$\mathrm{Z}$} & & $-1,15$ & $-0,16$ & $-1,53$ \\
\hline
\end{tabular}

O resultado obtido na comparação entre os escores dos problemas de comportamento apontaram um grupo de agressores bem reduzido e não foram encontrados resultados significativos neste sentido nem sob a ótica dos estudantes. Isto se deve talvez ao fato de que o termo problemas de comportamento apresenta pouca homogeneidade quanto à classificação, definição e diagnóstico (Peterson, 2009; Brioso \& Sarriá, 1995). Entretanto, Brioso e Sarriá (1995) concordam que problemas de comportamento envolvem desvio de comportamento social, isto é, comportamentos agressivos. Brioso e Sarriá (1995) assinalam também que os agressores com status de liderança tendem a possuir habilidades para antecipar o comportamento dos pares, sendo capazes de escolher a criança mais vulnerável, manipulando-a e tratando-a de forma negativa, porém não possuem capacidade de compartilhar a dor dos outros. Vale ressaltar que agressores de bullying costumam ser populares; tendem a envolver-se em uma variedade de comportamentos antissociais; vêm sua agressividade como qualidade; têm opiniões positivas sobre si mesmo e sentem prazer e satisfação em causar danos e sofrimentos a outros (Peterson, 2009). Por outro lado, um estudo identificou déficits importantes nas habilidades sociais de agressores (Garner \& Hilton, 2010). Os autores analisaram em seu estudo 77 crianças envolvidas no bullying. As análises mostraram que agressores apresentaram baixa autorregulação emocional comparando-se às vítimas. As dificuldades desses jovens referem-se a interpretar e responder adequadamente as emoções e de compreender as regras culturais. Desta forma, as hipóteses um e dois não foram confirmadas, o que pode estar relacionado ao fato de se ter encontrado uma população de agressores e vítimas pouco expressiva. Uma possível explicação para a não confirmação dos envolvidos no fenômeno foi a demonstração de preocupação quanto a avaliação que as outras pessoas poderiam fazer deles. Neste contexto, Thornberg (2010) afirma que especialmente a vítima decide ficar quieta, ou introverter-se, além de não se defender expressando o que sente de forma concisa. Thornberg (2010) complementa que os autores de bullying sofrem deterioração de sua escala de valores e, portanto, de 
seu desenvolvimento afetivo e moral. Desta forma, as crianças do presente estudo poderiam não ter ficado a vontade em suas respostas, ou estarem retraídas.

\section{Considerações finais}

A transição dos estudantes para o sexto ano do Ensino Fundamental traz situações que envolvem uma complexa relação entre o contexto social e acadêmico representado pelo processo de desenvolvimento pessoal. Essa transição exige compromisso com as atividades acadêmicas até a construção de novos conceitos de aprendizagem. Portanto, ao ampliar as relações sociais existe a possibilidade de que situações difíceis impliquem em tendências agressivas ou até mesmo a violência. A violência escolar está relacionada aos comportamentos agressivos e antissociais, incluindo os conflitos interpessoais, cujo tipo mais comum de violência praticada é o bullying entre outras. O presente estudo teve como principal intuito verificar se existe associação entre habilidades sociais e problemas de comportamento em agressores e vítimas de bullying. E mostrou que quanto mais problemas de comportamento os estudantes apresentam em situações consideradas difíceis no contexto escolar menor a proficiência de habilidades sociais. Deste modo considera-se que não houve diferença quanto as Habilidades Sociais nos envolvidos no bullying. Uma das limitações deste estudo encontra-se na baixa prevalência de bullying, ou seja, na sua reduzida amostra embora aceitável pela literatura por se tratar de uma amostra não probabilística. Também o processo de escolha das escolas e dos participantes que ocorreu por conveniência, sem avaliação prévia das demandas de prevalência sobre o bullying. A ausência do grupo classificado como envolvido (vítima e agressor) de forma expressiva pode ser considerada uma limitação, do contrário poderia apresentar-se com uma alternativa que possibilite avaliar os efeitos dessa relação.

Como perspectiva futura, se faz necessário que mais pesquisas sejam realizadas, na intenção de produzir mais sustentação acerca de quais as habilidades sociais auxiliam na diminuição do fenômeno bullying e que futuros trabalhos investigassem formas de identificação mais sensíveis desses envolvidos. Este estudo se propôs apresentar aporte para novos estudos sobre os temas abordados e estes auxiliem no enfrentamento dessa relação, seja na construção de materiais institucionais ou para programas de treinamento de professores.

\section{Referências}

Bandeira, M., Del Prette, Z. A. P., Del Prette, A., \& Magalhães, T. (2009). Validação das escalas de habilidades sociais, comportamentos problemáticos e competência acadêmica (SSRS-BR) para o ensino fundamental. Teoria e Pesquisa, 25(2), 271-282.

Bandeira, M. C., \& Hutz, C. S. (2012). Bullying: Prevalência, implicações e diferenças entre os gêneros. Psicologia Escolar e Educacional, 16(1), 35-44.

Barbosa, A. J. G. (2009). Questionário de Bullying. Juiz de Fora, MG: Autor. 
Barreto, S. O., Freitas, L. C., \& Del Prette, Z. A. P. (2011). Habilidades sociais na comorbidade entre dificuldades de aprendizagem e problemas de comportamento: Uma avaliação multimodal. Psico, 42(4), 503-510.

Brioso, A., \& Sarrià, E. (1995). Distúrbios de comportamento. In C. Coll, J. Palacios, \& A. Marchesi (Eds.), Desenvolvimento psicológico e educação: Necessidades educativas especiais e aprendizagem escolar (Vol. 3, pp. 157-168). Porto Alegre: Artes Médicas.

Brixval, C. S., Rayce, S. L., Rasmussen, M., Holstein, B. E., \& Due, P. (2012). Overweight, body image and bullying an epidemiological study of 11-to 15-years olds. The European Journal of Public Health, 22(1), 126-130.

Calbo, S. A., Busnello, F. D. B., Rigoli, M. M., Schaefer, L. S., \& Kristensen, C. H. (2009). Bullying na escola: Comportamento agressivo, vitimização e conduta pró-social entre pares. Contextos Clínicos, 2(2), 73-80.

Carpenter, D. E. \& Ferguson, C.(2011). Cuidado! Proteja seus filhos dos bullies. São Paulo: Batterfly

Centers for Disease Control and Prevention (CDC). (2011). Bullying among middle school and high school students: Massachusetts, 2009. MMWR. Morbidity and Mortality Weekly Report, 60(15), 465-471.

Cia, F., \& Costa, C. S. L. (2012). Desempenho acadêmico nas séries do ensino fundamental: Relação com o desenvolvimento social. Psicologia e Argumento, 30(68), 109-118. doi: 10.7213/psicolargum.5889

Del Prette, Z. A. P., \& Del Prette, A.(2009). Psicologia das Habilidades Sociais na Infância: Teoria e Prática. Rio de Janeiro. Vozes.

Del Prette, A., \& Del Prette, Z. A. P. (2010). Psicologia das relações interpessoais: Vivências para o trabalho em grupo. Petrópolis, RJ: Vozes.

Del Prette, Z. A. P., \& Del Prette, A. (2009). Avaliação de habilidades sociais: Bases conceituais, instrumentos e procedimentos. Psicologia das habilidades sociais: Diversidade teórica e suas implicações, 187-229.

Del Prette, Z. A. P., Barreto, S. D. O., \& Freitas, L. C. (2012). Habilidades sociais na comorbidade entre dificuldades de aprendizagem e problemas de comportamento: Uma avaliação multimodal. Psico, 42(4).

Garner, P. W., Hinton, T. S. (2010). Emotional display rules and emotion self-regulation: Associations with bullying and victimization in community-based after school programs. Journal of Community e Applied Social Psychology, 20(6), 480-96.

Gini, G., Pozzoli, T., \& Hauser, M. (2011). Bullies have enhanced moral competence to judge relative to victims, but lack moral compassion. Personality and Individual Differences, $50(5), 603-608$.

Gresham, F. M. (2009). Análise do comportamento aplicada às habilidades sociais. Psicologia das habilidades sociais: Diversidade teórica e suas implicações, 17-66. 
Hamiwka, L., Jones, J. E., Salpekar, J., \& Caplan, R. (2011). Child psychiatry. Epilepsy \& Behavior, 22(1), 38-46.

Lamas, K. C. A., Freitas, E. R., \& Barbosa, A. J. G. (2013). Bullying e Relação Professor-Aluno: Percepções de Estudantes do Ensino Fundamental. Psico, 44(2).

Malta,D.C.,Silva,M.A.I.,Mello, F.C.M.D., Monteiro, R.A., Sardinha, L.M.V., Crespo, C., .\& Porto, D.L. (2010). Bullying nas escolas brasileiras: resultados da Pesquisa Ncional de Saúde do Escolar (PeNSE), 2009. Ciencia Saúde Coletiva, 15(2), 3065-76.

Marchezini-Cunha, V., \& Tourinho, E. Z. (2010). Assertividade e autocontrole: Interpretação analítico-comportamental. Psicologia: Teoria e Pesquisa, 26(2), p. 295-304.

Matos,M.M.N.G. (2012). Prevenção da violência interpessoal em meio escolar: os professores, as famílias e a comunidade também marcam uma diferença?. Revista de Psiocologia da Criança e do Adolescente, 1(1),65-79.

Moura, D. R. D., Cruz, A. C. N., \& Quevedo, L. D. A. (2011). Prevalence and characteristics of school age bullying victims. Jornal de Pediatria, 87(1), 19-23.

Oliveira, J. C., \& Barbosa, A. J. G. (2012). Bullying entre estudantes com e sem características de dotação e talento. Psicologia: Reflexão e Crítica, 25(4), 747-755.

Olweus, D. (1989). Prevalence and incidence in the study of antisocial behavior: definitions and measurements. In Cross-national research in self-reported crime and delinquency (pp. 187-201). Springer Netherlands.

Pereira, B. O. (2008). Para uma escola sem violência: Estudo e prevenção das práticas agressivas entre crianças (2. ed.). Lisboa, Portugal: Ministério da Ciência e da Tecnologia.

Peterson, J. S. (2009). Myth 17: Gifted and talented individuals do not have unique social and emotional needs. Gifted Child Quarterly, 53(4), 280-282.

Thornberg, R. (2010). Schoolchildren's social representations on bullying causes. Psychology in the Schools, 47(4), 311-327.

Vanderbilt, D., \& Augustyn, M. (2010). The effects of bullying. Paediatrics and child Health, 20(7), 315-320.

Wiest, A. C., \& Lechner, G. (2013). Um olhar pedagógico sobre a transição da unidocência para a pluridocência. Revista Científica CENSUPEG, (1), p- 16.

Recebido/Received: 22/08/2015

Aprovado/Approved: 21/11/2015 